\title{
Optical nonlinearities of excitons in CuCl microcrystals
}

\author{
Yasuaki Masumoto, Makoto Yamazaki, and Hideyuki Sugawara \\ Institute of Physics, University of Tsukuba, Tsukubu, Ibaraki 305 , Jopan \\ (Received 16 March 1988; accepted for publication 10 August 1988)

\begin{abstract}
Nonlinear optical properties of excitons in $\mathrm{CuCl}$ microcrystals in $\mathrm{NaCl}$ host crystals were studied by the absorption saturation method. prominent absorption saturation of excitons was observed together with a biue shift. Optical nonlinearity was found to increase with an increase in the size of the $\mathrm{CuCl}$ microcrystals. The observed noninearities are very large compared with those in bulk $\mathrm{CnCl}$ crystals and GaAs quantum wells.
\end{abstract}

Recently optical properties of semiconductor microcrystals (quantum dots) have attracted much interest. This is because semiconductor microcrystais are expected to have novel optical properties in a way similar to semiconductor quantum weils. Also, semiconductor microcrystals are expected to have high optical nonlinearity. Therefore, they have a potential for becoming novel optoelectric devices which are useful in optical information processing. In this sense, the search for new materials including semiconductor microcrystals which have a high optical nonlinearity is important. So far, many authors have reported the guantum size effects for excitons in semiconductor microcrystals. Based on the classification made by Ekimov at ul, the quantum size effects are classified into two categories: electron or hole confinement and exciton confinement." Hanamura expects high optical nonlinearity for excitons in semiconductor microcrystals where exciton confinement takes place., The quantum size effect for excitons in CuCl microcrystals is a typical example of exciton confinement. Therefore, excitons in $\mathrm{CuCl}$ microcrystals are expected to give a high $\chi^{(3)}$ (the third-order nonlinear susceptibility). The purpose of this letter is to examine optical nonlinearity as a function of the size of $\mathrm{CuCl}$ microcrystals by means of nonlinear absorption, and to exarnine Hanamura's prediction.

Samples of $\mathrm{CuCl}$ microcrystais in $\mathrm{NaCl}$ host crystals are grown from high quality, zone-refned $\mathrm{CuCl}$ and vacum. distilled $\mathrm{NaCl}$. The transverse Bridgman method was used for the growth followed by various heat treatments. The heat treatment detemines the size distribution of $\mathrm{CuCl}$ microcrystals. We determined the size of the $\mathrm{CuCl}$ microcrystals by the absorption peak energy of $Z_{3}$ excitons at 77 I following the method established by Itoh. "The molar fraction of CuCl was determined by the inductively coupled piasma optical emission spectroscopy. The spectroscopy analyzes chemically the concentration ratio of $\mathrm{Cu}$ and $\mathrm{Na}$ ions. The five samples used in this study are listed in Table 1 . For the transmission experiments, samples were directly immersed in bubble-iree liquid nitrogen. We used an incandescent lamp for the weak-limit absorption experiments and a dye laser pumped by a nitrogen laser ( $600 \mathrm{~kW}, 10 \mathrm{~ms}$ ) for the nonlinear absorption experiments. A dye solution, BBQ in dioxane, was used as the active medium. The pulse duration was 7 ns and the puise energy was measured by using a pyroelectric energy detector (Gentec, EDIDOA and ED-X). The size of the excitation spot was measured under a microscope. The trunsmitted laser light was detected directly by a photomultiplier (Hamamatsu $R_{453}$ ) with a calibrated set of neutral density filters. A boxcar integrator was used for averaging. In the weak-limit absorption spectra, all the samples (15) show clear absorption peaks ascribed to $Z_{3}$ and $Z_{1,2}$ excitons at $77 \mathrm{~K}$. The absorption peak positions of $Z_{3}$ excitons of samples $2,3,4$, and 5 shift toward higher energies compared with the $Z_{3}$ exciton energy of bulk CuCl. Itoh studied both the $Z_{3}$ exciton energy and the size of $\mathrm{CuCl}$ microcrystals. The size was measured by means of small-angle $x$-ray scattering. On the basis of his study, the $Z_{3}$ exciton energy $E_{\mathrm{ex}}$ is best expressed by the formula

$$
E_{\mathrm{ex}}=E_{\mathrm{ex}}(\text { bulk })+(\pi h)^{2} /\left[2 M\left(a+0.5 a_{\mathrm{ex}}\right)^{2}\right] \text {, }
$$

where $E_{\mathrm{ex}}$ (bulk) means the $Z_{3}$ exciton energy of bulk $\mathrm{CuCl}$ crystals, $M\left(=2.3 m_{0}\right)$ is the $Z_{3}$ exciton translational mass, $a$ is the average radius of CuCl microcrystals, and $a_{\mathrm{cx}}(=0.68$ $n m)$ is the $Z_{3}$ exciton Bohr radius. The equation can give values of $a$ from the values of $E_{\mathrm{ex}}$. Thus the average radii of CuCl microcrystals in samples $2,3,4$, and 5 as determined are shown in Table 1. The shift of the $Z_{3}$ exciton absorption peak in sample 1 is much smaller than the absorption linewidth, so that the shift is not defniteiy obtained. Therefore, the radius of CuCl microcrystais in sample 1 is judged to be larger than $10 \mathrm{~nm}$.

In Fig. $\mathfrak{l}$, nonlinear absorption spectra around $\mathbb{Z}_{3}$ excitons in $\mathrm{CuCl}$ microcrystals are shown. The absorption coefficient $a$ is calculated by the formula $a=-\ln (T) / f d$, where $f$ is the volume fraction of $\mathrm{CuCl}$ in samples, $d$ is sample thickness, and $T$ is the transmittance of the iaser light intensity. The absorption spectra under the lowest densiry laser exciton almost agree with the weak-limit absorption spectra. With the increase of the exciration density, the absorption peak decreases prominently together with a biue shift. These nonlinear absorption features are similar to those observed in semiconductor quantum wells. ${ }^{5-8}$ The excitation intensiry

TA BLEI. List of samples. $d$ is the sample thickness, fis the volume fraction of $\mathrm{CuCl}$ in samples, and $E_{\mathrm{eK}}$ is the $Z_{3}$ exciton energy at $77 \mathrm{~K}$ observed in the absorption spectra. Size means the average radius of $\mathrm{CuCl}$ microcrystals.

\begin{tabular}{ccccc}
\hline $\begin{array}{c}\text { Sample } \\
\text { No. }\end{array}$ & $d(\mathrm{~mm})$ & $f(\%)$ & $E_{\mathrm{cx}}(\mathrm{eV})$ at $77 \mathrm{~K}$ & Size (nm) \\
\hline 1 & 0.44 & 0.12 & 3.219 & $\geqslant 10$ \\
2 & 0.34 & 0.12 & 3.223 & 5.7 \\
3 & 0.60 & 0.12 & 3.225 & 5.0 \\
4 & 0.55 & 0.12 & 3.236 & 3.3 \\
3 & 0.52 & 0.12 & 3.240 & 3.0 \\
\hline
\end{tabular}




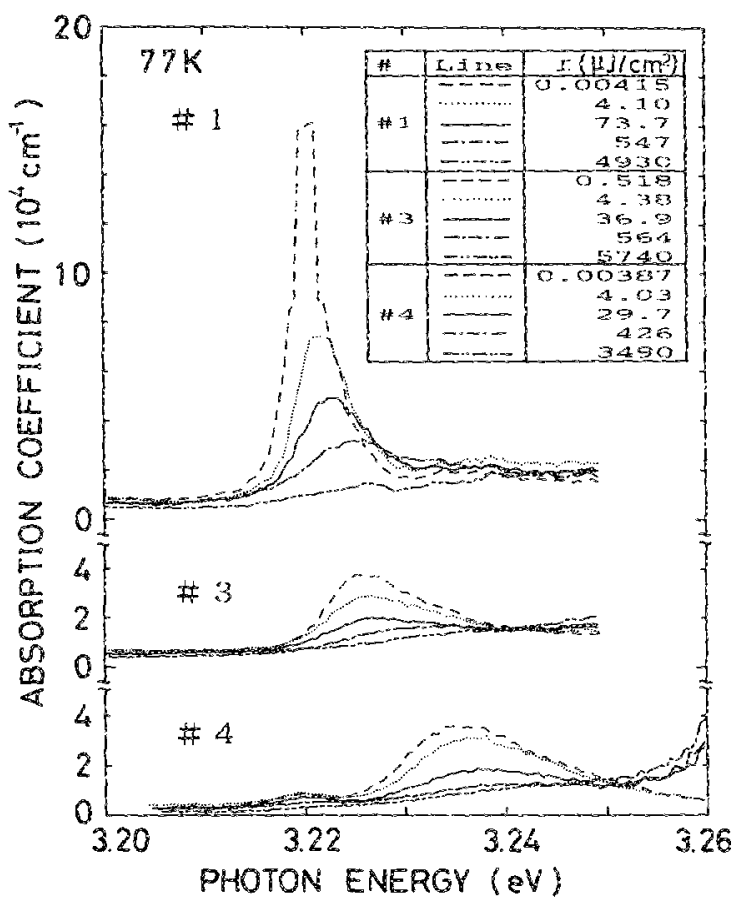

FIG. 1. Nonlinear absorption of $Z_{3}$ excirons in CuCl microcrystais in $\mathrm{NaCl}$ host crystals $(1,3$, and 4$)$. The sample temperature is $77 \mathrm{~K}$. The excitation densities corresponding to various fines are shown in the inset rable.

dependence of the absorption intensity is well described by the absorption saturation formula $\alpha=\alpha_{1} /\left(1+I / I_{s}\right)+\alpha_{2}$ (shown in Fig. 2), where $\alpha_{1}$ and $\alpha_{2}$ are fitting parameters and $l$ and $I_{s}$ are the laser light density and saturation density, respectively. In Fig. 2, various symbols indicate experimental points and lines least-squares-fitted resuits. The experimental points are well expressed by the formula except in the highest power density region. The saturation density thus obtained is plotted as a function of the size of the $\mathrm{CuCl} \mathrm{mi-}$ crocrystals in Fig. 3. Here, the $\mathrm{CuCl}$ microcrystals are assumed to be spheres and the size means the radius of the spheres, $a^{4}{ }^{4}$ Figure 3 shows that the saturation density de-

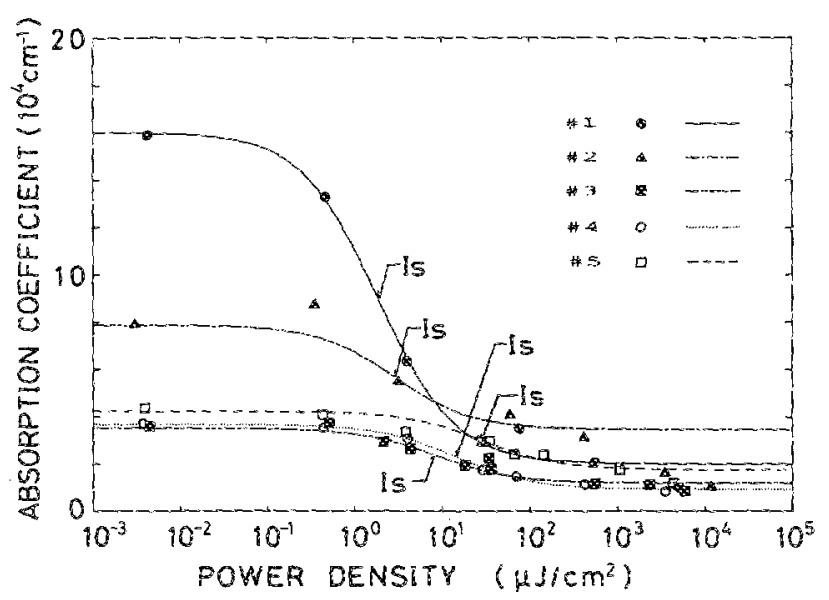

FIC. 2. Absorption coefficient $\alpha$ of $Z_{3}$ exciton absorption peak of samples (Nos. 1-5) as a function of laser power density 1 . Symbols show experinental values. Lines are least-squares fitted resuits by the equarion $\alpha==\alpha_{1} /$ $\left(1+I / I_{*}\right)+\alpha_{2}$, where $\alpha_{1}$ and $\alpha_{2}$ are fitting parameters. Salurated density is shown by $I_{s}$.

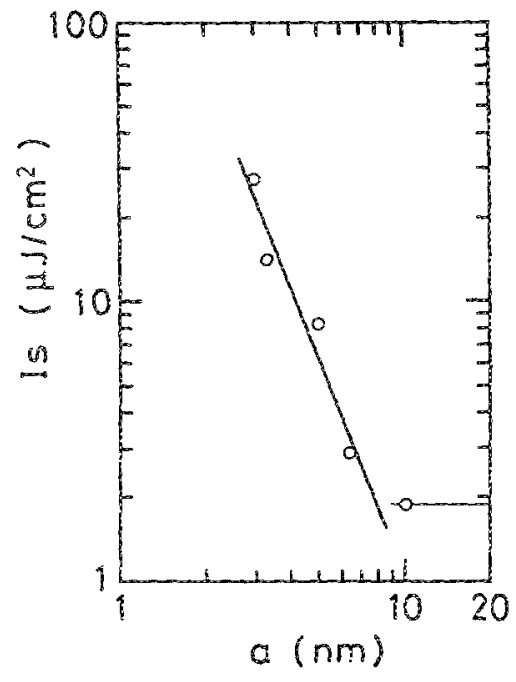

FIC. 3. Saturation density as a function of the average radius $a$ of the $\mathrm{CuCl}$ microcrystals. A solid line shows the $a^{-2.0}$ dependence.

creases with an increase of the size of the microcrystals. The relation between $I_{s}$ and $a$ is best expressed by $I_{s} \propto a^{-2.6}$. The saturation density $I_{s}$ can be related to the third-order nonlinear susceptibility $\chi^{(3)}$, when we consider the low-density excitation limit. The relation is written by

$$
\lim \chi^{(3)}=-\frac{\epsilon_{0} n_{0}^{2} c^{2} \alpha_{1} f d\left(\alpha_{1}+\alpha_{2}\right)}{\omega I_{s}\left\{1-\exp \left[-\left\{\alpha_{1}+\alpha_{2}\right) f d\right]\right\}},
$$

where $\epsilon_{0}$ is the dielectric constant in vacuum, $n_{0}$ the linear refractive index, and $c$ the light velocity in vacuum. ${ }^{9}$ The third-order nonlinear susceptibility $\chi^{(3)}$ increases with the increase of the size of CuCl microcrystals, because it is inversely proportional to $I_{s}$. The results almost agree with Hanamura's expectation of the $a^{3}$ dependence.

A more detailed analysis for sample 2 is shown in Fig. 4 , where we derive the experinental changes in the absorption $\Delta \alpha(\omega)$ and the corresponding refractive incex $\Delta n(\omega)$. The

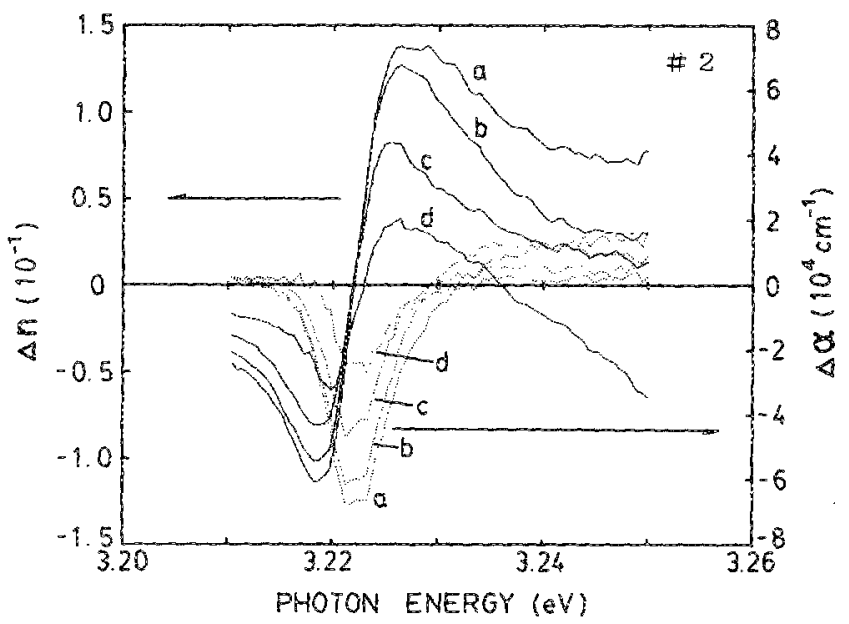

FIG. 4. Absorption change $\Delta \alpha(\omega)=\alpha(\omega, h)-\alpha\left(\omega, 3.1 \times 10^{-3} \mu \bar{H} / \mathrm{cm}^{2}\right)$ and the corresponding refractive index $\Delta n(\omega)$ for sample $2(a:=5.7 \mathrm{~nm})$. Dotted and solid lines show $\Delta \alpha(\omega)$ and $\Delta n(\omega)$, respectively. The excitation censities corresponding to $a, b, c$, and $d$ are $11800,3430,406$, and $3.19 \mu \mathrm{J} /$ $\mathrm{cm}^{2}$, respectively. 
change in the refractive index $\Delta n(\omega)$ is obtained by the Kramers-Kronig transformation ${ }^{10}$ :

$$
\Delta n(\omega)=\frac{c}{\pi} P \int_{0}^{\infty} \frac{d \omega^{\prime} \Delta \alpha\left(\omega^{\prime}\right)}{\omega^{\prime 2}-\omega^{2}} .
$$

Practicaly, we used the data of $\alpha(\omega, I)-\alpha(\omega$, $\left.3.1 \times 10^{-3} \mu J / \mathrm{cm}^{2}\right)$ for $\Delta \alpha(\omega)$. The change in the refractive index $\Delta n(\omega)$ is $4.0 \times 10^{-2}$ under an excitation density of 3.2 $\mathrm{HJ} / \mathrm{cm}^{2}\left(0.45 \mathrm{~kW} / \mathrm{cm}^{2}\right)$ and increases to $1.4 \times 10^{-1}$ under an excitation density of $11.8 \mathrm{~mJ} / \mathrm{cm}^{2}\left(1.7 \mathrm{MW} / \mathrm{cm}^{2}\right)$. The obtained $\Delta n(\omega)$ is larger than that observed in buk CuCL by two orders of magnitude. ${ }^{11}$ Figure 3 indicates that the saturation densities for samples 1 and 2 are $1.9 \mu 3 / \mathrm{cm}^{2}(270 \mathrm{~W} /$ $\left.\mathrm{cm}^{2}\right)$ and $2.9 \mathrm{\mu J} / \mathrm{cm}^{2}\left(420 \mathrm{~W} / \mathrm{cm}^{2}\right)$, respectively. The saturation density is smaller than that reported in GaAs quantum wels, ${ }^{7,12}$ indicating larger optical nonlinearity.

In conclusion, we experimentally demonstrated optical nonlinearity as a function of the size of $\mathrm{CuCl}$ microcrystals for the first time. Optical nonlinearity increases with an increase of the size of CuCl microcrystals. The observed nonlinearities are very high compared with those in bulk CuC: crystals and GaAs quantum wells. With a further increase of the size of CuCl microcrystals, a reduction of optical noninearity is expected. Testing the validity of this statement is necessary for a complete understanding of the optical nonlinearity due to excitons in $\mathrm{CuCl}$ microcrystals and remains to be studied in the future.

This work was supported in part by the Scientific Re- search Grant in Aid No. 62460022 from the Ministry of Education, Science and Culture of Japan.

'A. I. Ekimov, Al. L. Efros, and A. A. Onushchenko, Solid State Commun. $56,921(1985)$

${ }^{2} E$. Hannmura, Solid State Commun. 62, 465 (1987).

${ }^{3}$ E. Hatsamura, Phys. Rev. B 37, 1273 (1988).

${ }^{4} \mathrm{~T}$. Itoh, Solid State Phys. 23, 39 (1988) (in Japanese); T. Iroh, Y. Iwabuchi, and M. Kataoka, Phys. Status Solidi B 145, 567 (1988).

${ }^{5}$ N. P'eyghambarian, H. M. Gibbs, J. L. Jewell, A. Antonetti, A. Migus, D. Hulin, and A. Mysyrowicz, Phys. Rev. Lett. 53, 2433 (1984).

'Y. Masumoto, S. Shionoya, and H. Okamoto, Opt. Commun. 33, 385 (1985).

'Y. Masumoto, S. Tarucha, and HE. Okamoto, J. Phys. Soc. Jpn. 55, 57 (1986).

${ }^{8}$ D. Hulin, A. Mysyrowicz, A. Antonetti, A. Migus, W. T. Masselink, H. Morkoc, H. M. Gibbs, and N. Peyghambarian, Phys. Rey. B 33, 4389 (1986).

The relation is obtained as follows. The equation, $\alpha=\alpha_{1} /\left(1+I_{3}\right)$ $+\alpha_{2}$, is expanded to be $\alpha=\alpha_{1}\left(1-I / l_{s}\right)+\alpha_{2}$ in the weak limit of $l$. On the other hand, we can also obtain

$$
\alpha=\alpha_{1}+\alpha_{z}+\frac{\ln \chi^{(3)} \omega r\left\{1-\exp \left[-\left(\alpha_{1}+\alpha_{2}\right) f d\right]\right\}}{\epsilon_{0} n_{0}^{2} c^{2} f d\left(\alpha_{1}+\alpha_{2}\right)}
$$

by expanding Maruani's expression [see IEEE J. Quantum Electron. 18, $558(1980)]$. Then the $I$-dependent terms in both expressions are equated. ${ }^{15}$ D. A. B. Miller, C. T. Seaton, M. E. Prise, and S. D. Smith, Phys. Rev. Lett. 47, 197 (1981).

"The nonlinear refractive index $\Delta n$ around the exciton resonance in bulk CuCl is estimated to be $\sim 3 \times 10^{3}$ at an excitation density $400 \mathrm{~kW} / \mathrm{cm}^{2}$ at $3.2096 \mathrm{eV}$ on the basis of the data by Kuwata [J. Lumin. 38,247 (1987)]. ${ }^{12}$ D. A. B. Miller, D. S. Chemla, D. J. Eilenberger, P. W. Smith, A. C. Gossard, and W. T. Tsang, Appl. Phys. Lett. 41, 679 (1982). 